\title{
MONOPOLES, DUALITY, AND STRING THEORY
}

\author{
J. POLCHINSKI \\ Kavli Institute for Theoretical Physics, \\ University of California, \\ Santa Barbara, CA 93106-4030, USA \\ E-mail: joep@kitp.ucsb.edu
}

\begin{abstract}
Dirac showed that the existence of magnetic monopoles would imply quantization of electric charge. I discuss the converse, and propose two 'principles of completeness' which I illustrate with various examples. Presented at the Dirac Centennial Symposium, Tallahassee, Dec. 6-7, 2002.
\end{abstract}

\section{Theory}

It is a great honor to speak at this centennial of Paul Dirac. I also had the honor to speak at Pauli's centennial two years ago and at Heisenberg's last year, and on each occasion it has been interesting to go back and learn more about the work of these men. Of course the high point of their scientific lives came rather early, with the discovery of quantum mechanics, and the rest was anticlimax by comparison. But from a modern perspective the latter part is also fascinating, as they went on to confront many problems that are still timely today.

All three thought very hard about the divergences of quantum field theory, which was perhaps the central theoretical question of the day. On the other hand, Pauli and Heisenberg both looked for unified theories, while Dirac regarded this as premature. It was his conviction that the immediate problem facing theoretical physicists was to develop better mathematical tools: ${ }^{1}$

The most powerful method of advance [is] to perfect and generalize the mathematical formalism that forms the existing basis of theoretical physics.

Remarkably, not only did Dirac identify the necessary direction, he followed it successfully and provided many key ideas that continue to play a major role today: 
- Magnetic monopoles.

- Path integrals.

- Light cone dynamics.

- Membrane actions.

- Conformal and de Sitter symmetries.

- Constrained Hamiltonian dynamics.

- Canonical formulation of gravity.

For an anticlimax that is a pretty good career.

Dirac comes across in many ways as the first modern theoretical physicist. Many of his statements illustrate this, but the following strikes me as particularly apt: ${ }^{2}$

One must be prepared to follow up the consequences of theory, and feel that one just has to accept the consequences no matter where they lead.

Dirac is often quoted on the importance of mathematical beauty in one's equations; I did not choose one of these quotations because beauty is so difficult to define. He also made various statements that one should not being distracted by experiment; I did not choose one of these because they are inflammatory.

The reason that I find the chosen quotation so striking is that it is not supposed to be possible to follow theory alone. Without experimental guidance, it is said, one will quickly become lost. But of course today in high energy theory we are to a large extent following theory where it leads us, and we are rather confident that this is a correct and fruitful path. Why this approach can work is illustrated by Dirac's great discovery:

quantum mechanics + special relativity $\Rightarrow$ antiparticles .

This was not a direct deduction (though in the framework of quantum field theory, one can show that antiparticles are necessary for causality. ${ }^{3}$ ). Rather, when Dirac tried to find a consistent framework that combined quantum theory and special relativity, he found it very difficult — so much so that when he did find one he had great confidence in its inevitability, and was prepared to take its other consequences seriously.

Essentially, with the discovery of quantum mechanics and special relativity, and even more so with general relativity, theory has become very rigid, so that it is difficult to extend or modify our existing theories without making them inconsistent or otherwise unattractive. One reason for this is that relativity unifies space and time. For example, it seems almost inevitable that in quantum gravity space will be modified or cut off at short 
distance. One can imagine many sorts of modification to the structure of space, but it is much harder to alter the nature of time in a consistent way.

\section{The Necessity of Monopoles}

One of Dirac's remarkable discoveries was the connection between magnetic monopoles and charge quantization. ${ }^{1,4}$ Very early in the history of quantum theory, he recognized the important connection between geometry and quantum mechanics. Dirac showed that in the presence of a magnetic charge $g$, in order for the quantum mechanics of an electric charge $e$ to be consistent one had to have

$$
e g=2 \pi n
$$

Thus the existence of even a single magnetic charge forces every electric charge to be a multiple of $2 \pi / g$. From the highly precise electric charge quantization that is seen in nature, it is then tempting to infer that magnetic monopoles exist, and indeed Dirac did so: ${ }^{1}$

One would be surprised if nature had made no use of it.

I would like to discuss this from the point of view of the modern search for a unified theory, and to offer two general principles of completeness:

(1) In any theoretical framework that requires charge to be quantized, there will exist magnetic monopoles.

(2) In any fully unified theory, for every gauge field there will exist electric and magnetic sources with the minimum relative Dirac quantum $n=1$ (more precisely, the lattice of electric and magnetic charges is maximal).

Obviously neither of these is a theorem. Rather, they are aesthetic principles based on experience with a rather wide range of examples. I will give three examples of the first principle, and two of the second.

\subsection{Grand Unification}

The most well-known example of the first principle of completeness is the

't Hooft-Polyakov monopole. ${ }^{5}$ If the $U(1)$ of electromagnetism is embedded in a semisimple group, for example in grand unification

$$
S U(3) \times S U(2) \times U(1) \subset S U(5),
$$

then electric charge is necessarily quantized, since it descends from the quantized representations of the unified group. Under precisely these con- 
ditions, 't Hooft and Polyakov showed that magnetic monopoles will exist as smooth but topologically nontrivial classical solutions.

Let me give a brief description of this idea. Dirac showed that the vector potential for a magnetic charge had a singularity along a string extending from the charge. Figure 1 shows a Dirac string. Let us parallel transport a

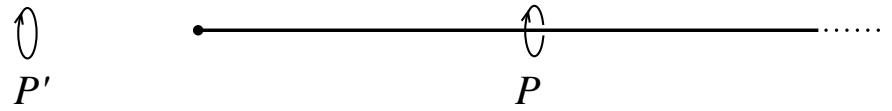

Figure 1. A Dirac string extending from the monopole.

charged field $\psi$ on the infinitesimal path $P$ around it according to

$$
d \psi=i e \vec{A} \cdot \vec{d} x \psi
$$

Because of the string singularity, the field picks up a net phase $e g$ in the process; this is unobservable precisely if $e g=2 \pi n$. One can think of the phase of $\psi$ as looping around the $U(1)$ group, which is a circle, $n$ times. Now, if we pull the loop off of the string to the position $P^{\prime}$, the field is nonsingular and the phase is constant. Hence the net phase must drop rapidly from $2 \pi n$ to zero as the loop is pulled past the monopole, and this observable phase signifies a singularity in the field. When $U(1)$ is embedded in a semisimple group, however, the loop can become topologically trivial: in $S U(2)$ for example, a rotation through $4 \pi$ can be smoothly deformed to a trivial path. Thus there are smooth field configurations, which at long distance look like Dirac monopoles, with a net $U(1)$ magnetic charge.

As a postscript to the talk, I should note that this argument does not require that one can obtain $e g=2 \pi$, and indeed this depends on the field content. In the Georgi-Glashow model $U(1) \subset S U(2)$, if there are only fields of integer isospin then the minimum quantum is $e g=4 \pi$, while if there are fields of half-integer isospin then $e g=2 \pi$ is obtained. (This is related to the fact that some simple groups do have a finite set of nontrivial closed loops.) So in the sense of the second principle I would have to say that this theory is not fully unified, precisely because its matter content is not fixed but subject to arbitrary choice.

\subsection{Kaluza-Klein Theory}

If spacetime is five-dimensional, with the added dimension $x^{4}$ being periodic, then five-dimensional gravity gives rise to both gravity and a Maxwell 
field in four dimensions. This was perhaps the first application of spontaneous symmetry breaking as a unifying concept - the laws of physics are invariant under Lorentz transformations in all five spacetime dimensions, while the state we live in is invariant only under the four-dimensional symmetry group. The metric components $g_{\mu 4}$ become the Maxwell potential, and gauge invariance arises from reparameterizations of $x^{4}$. What the fourdimensional physicist sees as electric charge is therefore momentum in the 4-direction, and it is quantized because of the periodicity in this direction.

A Dirac monopole configuration would again have a string, which is now a coordinate singularity as in Figure 1. An infinitesimal loop in the fivedimensional geometry, whose projection to four dimensions is the loop $P$, makes one or more circuits of the $x^{4}$ coordinate. An infinitesimal loop away from the string, such as $P^{\prime}$, does not loop the $x^{4}$ direction. Thus there is again singular behavior as the loop is pulled past the monopole. However, Gross, Perry, and Sorkin ${ }^{6}$ showed that again there are smooth geometries that look like Dirac monopoles outside of some core region. The point is that if the radius of the $x^{4}$ direction shrinks to zero in an appropriate way, then at the origin there is only a coordinate singularity and the loop can be smoothly slid off the string.

\subsection{U(1) Lattice Gauge Theory}

The final example of the first principle is rather different from the others, but very vividly illustrates the connection between charge quantization and magnetic monopoles. When one puts $U(1)$ gauge theory on a spatial or spacetime lattice, the basic variables $A_{l}$ live on links, directed pairs of adjacent points. One can think of $A_{l}$ as the lattice approximation to $\int \vec{A} \cdot d \vec{x}$, integrated from one site to the next.

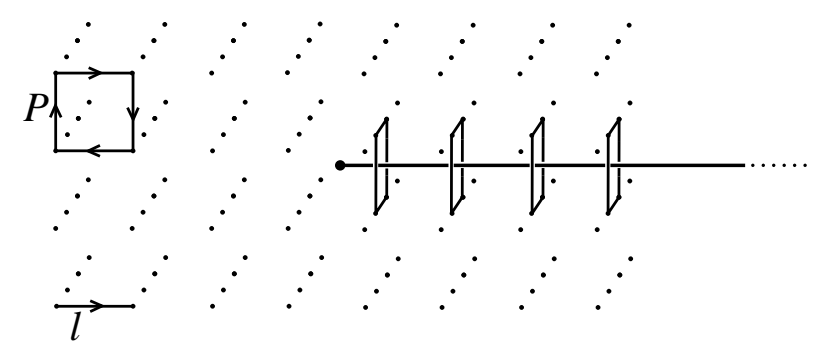

Figure 2. Part of a spatial lattice. A typical link $l$ and plaquette $P$ are illustrated. A magnetic monopole and its Dirac string are shown, hidden between the sites of the lattice. 
There are two versions of $U(1)$ lattice gauge theory, according to whether $A_{l}$ is a periodic variable or takes values on the whole real line:

$$
\begin{aligned}
\text { compact theory: } & A_{l} \cong A_{l}+2 \pi, \\
\text { noncompact theory: } & -\infty<A_{l}<\infty .
\end{aligned}
$$

For example these theories have different actions. The analog of the field strength is the sum of the link variables around a plaquette,

$$
F_{P}=\sum_{l \in P} A_{l}
$$

In the noncompact case the simplest action would be the sum over plaquettes of $F_{P}^{2}$, while in the compact case it would be the sum of $\left(1-\cos F_{P}\right)$. Alternately, one can describe the compact theory entirely in terms of $U_{l}=e^{i A_{l}}$, and the action is constructed from $U_{P}=\prod_{l \in P} U_{l}$.

In the noncompact case charge is not quantized, one can transport any charge $e$ from one site to the next with the phase $e^{i e A_{l}}$. However, in the compact case this is defined only for $e$ an integer, and so charge is quantized (we are working here in units where the charge is dimensionless and the minimum value is 1 , but we can rescale $A_{l}$ to other systems of units). Precisely in keeping with the general principle, one finds that there are magnetic monopoles in the compact case but not the noncompact one. The Dirac string is a line of plaquettes on all of which $F_{P} \approx-1($ or $-n)$, while it is $\ll 1$ on all other plaquettes except those very close to the monopole. The line ends at the monopole. From the definition of $F_{P}$ it follows that $F_{P}$ summed over any closed surface is zero, so if we consider a large surface surrounding the monopole the sum of all the small fluxes must be +1 (or $+n)$ to cancel the contribution of the string: this is the monopole flux. Nothing is singular, everything is made finite by the lattice. In the compact theory the string costs no energy because $U_{P}$ is everywhere near unity, while in the noncompact theory the string is visible to noninteger charges and has an energy proportional to its length.

This example makes vividly clear the connection between charge quantization and the existence of monopoles. In fact, the compact theory can be rewritten as the noncompact theory coupled to a magnetic monopole field. For some reviews of this subject see Ref. 4 .

\subsection{The Kalb-Ramond Field}

Many supergravity theories have an antisymmetric tensor field $B_{\mu \nu}$, with a generalized form of gauge invariance

$$
\delta B_{\mu \nu}=\partial_{\mu} \lambda_{\nu}-\partial_{\nu} \lambda_{\mu}
$$


If we consider such a theory in a Kaluza-Klein geometry, the components $B_{\mu 4}$ again become a four-dimensional Maxwell theory. However, unlike the Maxwell field from the metric, there are no states in supergravity that are electrically charged under this gauge field - there is no way to minimally couple any field to $B_{\mu \nu}$ so as to give rise to a minimal coupling to $B_{\mu 4}$.

This incompleteness is not an inconsistency, but it is somewhat puzzling and unattractive that there is this asymmetry between the gauge fields from the metric and those from $B_{\mu \nu}$. In this respect string theory completes supergravity. The two-form $B_{\mu \nu} d x^{\mu} d x^{\nu}$ can be integrated over the worldsheet of the string, just as $A_{\mu} d x^{\mu}$ can be integrated over the world-line of a particle, and just such a coupling is present in string theory. If a string wraps around the periodic $x^{4}$ direction, the integral $d x^{4}$ produces a coupling to $B_{\mu 4}$. Thus the string winding states are electrically charged.

There should also be a corresponding magnetic source. We can discuss this in four dimensions, but it is clearer to start in the full ten-dimensional theory. In general a $p$-dimensional object (brane) couples to a $(p+1)$-form potential, through the integral

$$
\int B_{\mu_{1} \ldots \mu_{p+1}} d x^{\mu_{1}} \ldots d x^{\mu_{p+1}}
$$

over the world-history of the brane. The curl of this potential gives a $(p+2)$-form field strength. Contracting this with the spacetime $\epsilon$ tensor gives a dual $(D-p-2)$-form field strength, where $D$ is the dimension of spacetime. This corresponds to a $(D-p-3)$-form magnetic potential, and so couples magnetically to a $p^{\prime}$-dimensional object for $p^{\prime}=D-p-4$. In other words, $p+p^{\prime}=D-4$. The familiar electric and magnetic charges are simply $p=p^{\prime}=0$ in $D=4$, but the Dirac quantization argument extends directly to all such pairs. ${ }^{7,8}$

For the case at hand $D=10$ and $p=1$ and so $p^{\prime}=5$ : the magnetic object is a five-brane. This was found by Callan, Harvey, and Strominger ${ }^{9}$ as a solitonic solution to the low energy field theory of string theory it is the Neveu-Schwarz (NS) five-brane. Curiously their first paper found a five-brane charge of 8 Dirac units. This is consistent, but rather odd. Shortly afterward the authors realized that they had used inconsistent normalizations of $B_{\mu \nu}$, and found that the magnetic charge is the minimum Dirac quantum.

\subsection{D-Branes}

The final example has great significance for me. In string theory, in addition to the NS-NS field $B_{\mu \nu}$ there are other 'Ramond-Ramond' form potentials. 
The terminology refers to the fact that a closed string state is the product of the states of its right- and left-moving oscillations, so one can make a bosonic field out of two bosonic states (these are called NS-NS) or out of two fermionic states (called R-R). Unlike $B_{\mu \nu}$ these forms do not couple to the fundamental string.

However, string theory has extended objects of a distinctive type, the Dbranes. These are like topological defects, with the notable property that a (normally closed) string can end on them. There were various early discussions of strings with such fixed endpoints. In particular it was argued that these objects had to be included in string theory because one encountered them if one took the normal open string theory and followed it to small radius ( $T$-duality). For some time afterward these objects were still regarded as curiosities, but in the wake of string duality, in which R-R charges are necessary to fill out the multiplets, it was realized ${ }^{10}$ that the consistency of weak/strong duality and $T$-duality required that the D-branes carry the R-R charge. Further, they exist with just the right dimensions to provide a full set of electric and magnetic sources, even for nondynamical 9- and 10-form fields.

The calculation of $e g$ is interesting. It is shown schematically in Figure 3. This process can be regarded as the emission and absorption of a closed string, giving rise to a force between the two D-branes. It can equally be regarded as a vacuum loop of an open string, and this is by far the most direct way to calculate it, by summing the zero point energies of open string modes. From the sum one reads off the potential, which gives the coupling of the D-branes to the various forms. Note that unlike the calculations of the charges of the various other objects that have been discussed there is nothing obviously topological about this, it is essentially the calculation of a Casimir energy. In particular there is no reason that it should give anything like an integer for $e g$. Indeed, the first attempts at the calculation gave undesirable powers of $\pi$ and $1 / 2$, but after a day of debugging the result is that the product of the charge of a $\mathrm{D} p$-brane and that of a $\mathrm{D}(6-p)$-brane is exactly $2 \pi$.

As a postscript, I should note that in any theory with gravity one can make electric and magnetic sources trivially, by having the field lines end on a black hole singularity (the R-R charged objects were first described in this form $\left.{ }^{11}\right)$. In this description there is no direct way to determine the actual spectrum of charges. However in string theory we can usually turn off the gravitational force by turning down the coupling, so that the black hole goes over to something nonsingular, and in this way we know the spectrum. 


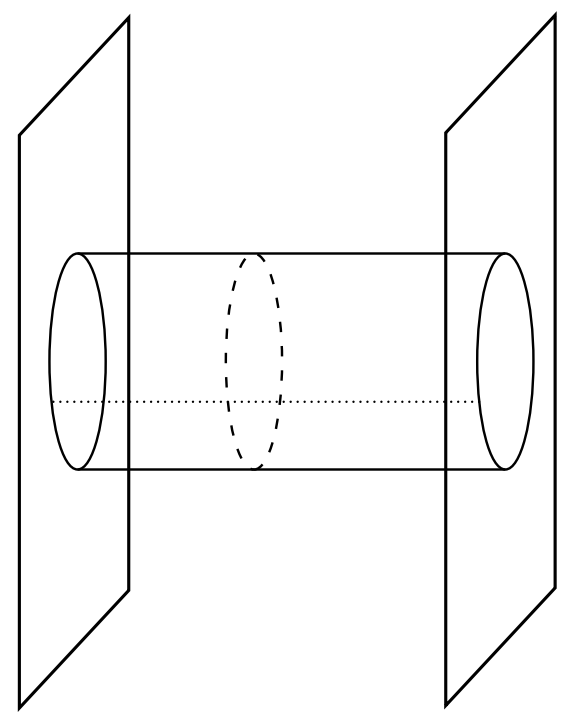

Figure 3. Two D-branes (the vertical planes) and a cylindrical string world-sheet with one boundary on each D-brane. The world-sheet can be regarded either as an open string with one end on each D-brane (the dotted line) traveling in a vacuum loop, or a closed string (the dashed line) emitted by one D-brane and absorbed by the other.

\section{Conclusions}

\subsection{The Existence of Monopoles}

By the end of his career Dirac became less certain about the existence of monopoles. He forgot his earlier dictum to ignore experiment! But as I have discussed, the existence of magnetic monopoles seems like one of the safest bets that one can make about physics not yet seen. It is very hard to predict when and if monopoles will be discovered. If their mass is at the grand unified scale as one expects, then they will be beyond the reach of accelerators, while inflation has almost certainly diluted any primordial monopoles beyond discovery. It is curious to contemplate this unfortunate situation, where theory predicts the existence of an object (and its production, but in experiments that can only be carried out in thought) and at the same time suggests that it may never be seen. But we must continue to hope that we will be lucky, or unexpectedly clever, some day.

\subsection{Duality and Beauty}

Many of the electric and magnetic objects that I have discussed look quite different from one another, but there is strong evidence that in each case 
that there are dualities that interchange them. For Dirac this would have been a triviality. He notes ${ }^{4}$ that his theory of pointlike electric and magnetic charges is invariant under the interchange of the two objects, along with the interchange of electric and magnetic potentials. Dirac's theory is rather formal, since the magnetic coupling is the inverse fine structure constant, but one can regard the lattice as providing a precise definition of a cutoff theory, and with appropriate choice of the action it is self-dual. ${ }^{7}$

For the grand unified and Kaluza-Klein cases, however, any duality must be quite nontrivial. In these cases the electric charges are pointlike quanta, while the magnetic charges are smooth classical configurations. To be precise, this is the picture at weak coupling. Now it would not be surprising that as the coupling is turned up the electric objects begin to emit pairs and become big and fuzzy like the solitons. The great surprise (duality) is that when the coupling becomes very large the magnetic objects become more and more pointlike and the theory can be described in terms of their local fields. It is a remarkable property of the quantum theory that the degrees of freedom can, at least with some assistance from supersymmetry, reorganize themselves in this way. Indeed, we do not fully understand the details of this, but the number of independent consistency checks is enormous.

Even further, in string theory all of the electric and magnetic objects that have been discussed here, with the (possible ${ }^{12}$ ) exception of the lattice examples, are related to one another by dualities. These examples involve widely different aspects of gauge field geometry, spacetime geometry, 'stringy' geometry, string perturbation theory, and quantum and classical physics. The existence of a single structure that unifies such a broad range of physical and mathematical ideas, and many others as well, is unexpected and remarkable. Earlier I declined to define beauty, but one can recognize it when one sees it, and here it is. This is one illustration of why the scientific path that Dirac laid out has been such a fruitful one in recent times.

\section{Acknowledgments}

This work was supported by National Science Foundation grants PHY9907949 and PHY00-98395.

\section{References}

1. P. A. Dirac, "Quantised Singularities In The Electromagnetic Field," Proc. Roy. Soc. Lond. A 133, 60 (1931).

2. P. A. Dirac, 1977 Varenna lecture, quoted by M. Jacob in A. Pais, M. Ja- 
cob, D. I. Olive, and M. F. Atiyah, "Paul Dirac: The Man and his Work," Cambridge, UK: Univ. Pr. (1998).

3. See for example Chapter 2 of M. E. Peskin and D. V. Schroeder, "An Introduction To Quantum Field Theory," Reading, USA: Addison-Wesley (1995).

4. P. A. Dirac, "The Theory Of Magnetic Poles," Phys. Rev. 74, 817 (1948).

5. G. 't Hooft, "Magnetic Monopoles In Unified Gauge Theories," Nucl. Phys. B 79, 276 (1974);

A. M. Polyakov, "Particle Spectrum In Quantum Field Theory," JETP Lett. 20, 194 (1974) [Pisma Zh. Eksp. Teor. Fiz. 20, 430 (1974)].

6. D. J. Gross and M. J. Perry, "Magnetic Monopoles In Kaluza-Klein Theories," Nucl. Phys. B 226, 29 (1983);

R. d. Sorkin, "Kaluza-Klein Monopole," Phys. Rev. Lett. 51, 87 (1983).

7. M. E. Peskin, Annals Phys. 113, 122 (1978);

R. Savit, "Duality In Field Theory And Statistical Systems," Rev. Mod. Phys. 52, 453 (1980).

8. P. Orland, "Instantons And Disorder In Antisymmetric Tensor Gauge Fields," Nucl. Phys. B 205, 107 (1982);

R. I. Nepomechie, "Magnetic Monopoles From Antisymmetric Tensor Gauge Fields," Phys. Rev. D 31, 1921 (1985);

C. Teitelboim, "Monopoles Of Higher Rank," Phys. Lett. B 167, 69 (1986).

9. C. G. Callan, J. A. Harvey and A. Strominger, "Worldbrane Actions for String Solitons," Nucl. Phys. B 367, 60 (1991); "World Sheet Approach To Heterotic Instantons And Solitons," Nucl. Phys. B 359, 611 (1991).

10. J. Polchinski, "Dirichlet-Branes and Ramond-Ramond Charges," Phys. Rev. Lett. 75, 4724 (1995) [arXiv:hep-th/9510017].

11. G. T. Horowitz and A. Strominger, "Black Strings and P-Branes," Nucl. Phys. B 360, 197 (1991).

12. S. Hellerman, "Lattice Gauge Theories have Gravitational Duals," arXiv:hepth/0207226. 